\title{
ERRATUM TO "QUASICONFORMAL HARMONIC MAPPINGS WITH THE CONVEX HOLOMORPHIC PART"
}

\author{
Dariusz Partyka, Ken-ichi Sakan and Jian-Feng Zhu
}

The John Paul II Catholic University of Lublin, Institute of Mathematics and Computer Science

Al. Racławickie 14, P.O. Box 129, 20-950 Lublin, Poland; partyka@kul.lublin.pl and The State School of Higher Education in Chełm, Institute of Mathematics and Information Technology, Pocztowa 54, 22-100 Chełm, Poland

Osaka City University, Graduate School of Science, Department of Mathematics

Sugimoto Sumiyoshi-ku, Osaka, 558-8585, Japan; ksakan@sci.osaka-cu.ac.jp

Huaqiao University, School of Mathematical Sciences

Quanzhou-362021, P. R. China; flandy@hqu.edu.cn

Corollary 2.7. Given $R>0$ let $H$ be a conformal mapping in $\mathbf{D}$ such that $H(\mathbf{D})$ is a convex domain and $\mathbf{D}(H(0), R) \subset H(\mathbf{D})$. Then $H$ is co-Lipschitz with

$$
\mathrm{L}^{-}(H)=\mathrm{D}^{-}(H) \geq \frac{R}{4} \text {. }
$$

Proof. Under the assumption of the corollary we see that the mapping $\mathbf{D} \ni z \mapsto$ $\tilde{H}(z):=H(z)-H(0)$ maps $\mathbf{D}$ onto a convex domain and $\mathbf{D}(0, R) \subset \tilde{H}(\mathbf{D})$. Since $\tilde{H}(0)=0$ we conclude from [10, Corollary 3.1] (see also [5, Theorem 2.5]) that

$$
\left|H^{\prime}(z)\right|=\left|\tilde{H}^{\prime}(z)\right| \geq \frac{R}{4}, \quad z \in \mathbf{D} .
$$

Therefore $\mathrm{D}^{-}(H) \geq R / 4$, and so the inequality in (2.19) holds. By Remark 2.6 we see that the equality in (2.19) holds. Therefore $H$ is a co-Lipschitz mapping, which is our claim. in $\mathbf{D}$,

Let us consider the following deformations of a harmonic mapping $F=H+\bar{G}$

$$
\mathbf{D} \ni z \mapsto F_{\varepsilon}(z):=H(z)+\varepsilon \overline{G(z)}, \quad \varepsilon \in \mathbf{C} .
$$

Using now the decomposition (2.2) we derive the following theorem.

Theorem 2.8. Let $F=H+\bar{G}$ be a sense-preserving harmonic mapping in D. Suppose that $H$ is injective, $H(\mathbf{D})$ is a rectifiably $M$-arcwise connected domain with a given $M \geq 1$ and that $F$ is not a conformal mapping. Then for every $\varepsilon \in$ $\mathbf{D}\left(1 / M\left\|\mu_{F}\right\|_{\infty}\right), F_{\varepsilon}$ is a quasiconformal harmonic mapping. Moreover, if $M=1$, then $F_{\varepsilon}$ is co-Lipschitz.

Proof. Fix $\varepsilon \in \mathbf{D}\left(1 / M\left\|\mu_{F}\right\|_{\infty}\right)$. By setting $H(\mathbf{D}) \ni z \mapsto \phi(z):=\bar{\varepsilon} G \circ H^{-1}(z)$, we see that for every $z \in H(\mathbf{D})$,

$$
\left|\phi^{\prime}(z)\right|=\left|\bar{\varepsilon} \frac{G^{\prime}\left(H^{-1}(z)\right)}{H^{\prime}\left(H^{-1}(z)\right)}\right|=|\varepsilon|\left|\mu_{F}\left(H^{-1}(z)\right)\right| \leq|\varepsilon|\left\|\mu_{F}\right\|_{\infty} .
$$

https://doi.org/10.5186/aasfm.2018.4355

2010 Mathematics Subject Classification: Primary 30C62, 30C55.

Key words: Harmonic mappings, quasiconformal mappings, Lipschitz condition, bi-Lipschitz condition, co-Lipschitz condition, Jacobian. 
Hence $M \mathrm{D}^{+}(\phi) \leq M|\varepsilon|\left\|\mu_{F}\right\|_{\infty}<1$. From Lemma 2.4 it follows that $\mathrm{I}[\phi]$ is biLipschitz, and so $\mathrm{I}[\phi]$ is quasiconformal. Since $F_{\varepsilon}=\mathrm{I}[\phi] \circ H, F_{\varepsilon}$ is a quasiconformal mapping as a composition of quasiconformal ones. Suppose now that $M=1$, i.e., $H(\mathbf{D})$ is a convex domain. By the conformality of $H, \mathbf{D}(H(0), R) \subset H(\mathbf{D})$ for a certain positive number $R$. Then by Corollary 2.7 we see that $H$ is a co-Lipschitz mapping. Therefore $F_{\varepsilon}$ is a co-Lipschitz mapping as a composition of co-Lipschitz ones, which proves the theorem.

\section{References}

[5] KalaJ, D.: On harmonic diffeomorphisms of the unit disc onto a covex domain. - Complex Variables 48:2, 2003, 175-187.

[10] Partyka, D., and K. SAKAn: On a variant of Heinz's inequality for harmonic mappings of the unit disk onto bounded convex domains. - Bull. Soc. Sci. Lett. Łódź Sér. Rech. Déform. 59:2, 2009, 25-36.

[PSZ] PARTYKA, D., K. SAKAn, and J.-F. Zhu: Quasiconformal harmonic mappings with the convex holomorphic part. - Ann. Acad. Sci. Fenn. Math. 43:1, 2018, 401-418.

Received 6 April 2018 • Accepted 6 April 2018 